\title{
Solution of simple toy models via thimble regularization of lattice field theory
}

\section{Giovanni Eruzzi* and Francesco Di Renzo}

University of Parma and INFN

E-mail: giovanni.eruzzi@pr.infn.it, francesco.direnzo@pr.infn.it

\begin{abstract}
The sign problem prevents lattice simulations of many models of physical interest. One proposal for evading such problems is the thimble regularization. In this proceeding we discuss its application to the $\phi^{4} 0$-dimensional integral, which is a toy model that has been studied for many years as a prototype of complex-valued action and raises several problems within a treatment via complex Langevin; this model can be successfully solved with the thimble approach, though it exhibits a very non trivial structure. We discuss the details of the thimble structure in this model along with three different algorithms that we have used to get precise numerical results.
\end{abstract}

The 32nd International Symposium on Lattice Field Theory

23-28 June, 2014

Columbia University New York, NY

\footnotetext{
* Speaker.
} 


\section{Motivation}

The sign problem, which affects many field theories at finite chemical potential, is due to the presence of complex terms in the action; this makes it impossible to perform Monte Carlo simulations using $\exp (-S)$ as a probability weight. One of the approaches one can employ in this case is reweighting, but this cannot be used in all regions in parameter space because of the oscillatory part of the action $S^{I}=\mathfrak{I}(S)$ scaling exponentially with the volume of the system. The main motivation behind the thimble approach is that integrating along the thimble automatically keeps $S^{I}$ constant, thus removing the sign problem completely. In the framework of the thimble approach, it is interesting to study 0-dimensional field theories, that is complex integrals; these integrals, although simple as well as analytically computable, feature a non-trivial thimble structure and are often problematic for complex Langevin simulations. The main topic of this contribution will be a review of complex Morse theory applied to the one-dimensional case and a detailed study of the 0 -dimensional $\phi^{4}$ toy model. We show that we can do Monte Carlo simulations for this model and the sign problem is completely under control.

\section{Picard-Lefschetz (complex Morse) theory: a primer for 0-dimensional toy models}

In this brief introduction to complex Morse theory (also known as Picard-Lefschetz theory), we focus on the fundamental aspects which are relevant for the present discussion of the 0-dimensional $\phi^{4}$ model. We follow [1] for the notation, keeping in mind that the formulas can be easily extended to the case of quantum field theories with many degrees of freedom. Let us say we have some action $S$ and we want to calculate the expectation value of some observable which is a (holomorphic) function $f$ of the only degree of freedom of the 0 -dimensional theory, the real "field" $\phi$. The action is a complex holomorphic function of this field and we set $S=S^{R}+i S^{I}$, while the computation of the expectation value is carried on along a given integration cycle $\mathscr{C}$. In our model, we simply set $\mathscr{C}=\mathbb{R}$ and we are interested in correlators such as

$$
\left\langle\phi^{n}\right\rangle=\frac{1}{Z} \int_{\mathbb{R}} \mathrm{d} \phi \phi^{n} e^{-S(\phi)}
$$

with the partition function given by

$$
Z=\int_{\mathbb{R}} \mathrm{d} \phi e^{-S(\phi)}
$$

Morse theory states that integrals such as that in (2.1) can be decomposed as a sum of integrals along Lefschetz thimbles $\mathscr{J}_{\sigma}$ associated with the critical points of the action in the complex plane (i.e. the field has been complexified)

$$
\left\langle\phi^{n}\right\rangle=\frac{1}{Z} \sum_{\sigma} m_{\sigma} \int_{\mathscr{J}_{\sigma}} \mathrm{d} \phi \phi^{n} e^{-S(\phi)}
$$

where we call the complexified field $\phi=x+i y$. The $m_{\sigma}$ are integer coefficients with sign and their value is thoroughly discussed below for the $\phi^{4} 0$-dimensional model. Each of these integrals is guaranteed to be convergent and they also share the striking property that along them the imaginary 
part of the action remains constant, so the sign problem due to the imaginary part of the action is avoided. Now let us call $\left\{\phi_{\sigma}\right\}$ the set of all the action critical points (which are assumed to be non-degenerate). As $S^{I}$ stays constant along the thimbles, formula (2.3) can be rephrased as

$$
\left\langle\phi^{n}\right\rangle=\frac{1}{Z} \sum_{\sigma} m_{\sigma} e^{-i S^{I}\left(\phi_{\sigma}\right)} \int_{\mathscr{J}_{\sigma}} \mathrm{d} \phi \phi^{n} e^{-S^{R}(\phi)}
$$

which renders the integration over a real Boltzmann weight manifest. Let us now see how integration along the thimbles is implemented. The stable thimble $\mathscr{J}_{\sigma}$ associated with the critical point $\phi_{\sigma}$ is defined as the curve in the $\phi$ complex plane given by those solutions of the equations of steepest ascent, that is

$$
\left\{\begin{array}{l}
\frac{\mathrm{d} x}{\mathrm{~d} t}=+\frac{\partial S^{R}(x, y)}{\partial x} \\
\frac{\mathrm{d} y}{\mathrm{~d} t}=+\frac{\partial S^{R}(x, y)}{\partial y}
\end{array}\right.
$$

that start from the critical point $\phi_{\sigma}$ for $t \rightarrow-\infty$. To recover these curves, in practice one integrates the equations (2.5) starting near the critical point $\phi_{\sigma}$ for an arbitrarily long time $t$, provided that the starting direction is choosen correctly: the direction (in the $x y$ plane) of the thimble $\mathscr{J}_{\sigma}$ near the critical point is given by the eigenvector of positive eigenvalue of the hessian matrix of $S^{R}(x, y)$ computed at the critical point (by holomorphicity the hessian has two eigenvalues opposite in sign). By using the steepest ascent equations and the chain rule, it is easy to show that $S^{R}$ always increases along the flow, so our integration, as $t \rightarrow+\infty$, brings $\exp \left(-S^{R}\right)$ to 0 , thus ensuring convergence of the integrals along the thimble. Associated with a given critical point $\phi_{\sigma}$ there is also an unstable thimble $\mathscr{K}_{\sigma}$, which can be recovered by the same procedure described above, but with the eigenvector of the hessian of $S^{R}$ with negative eigenvalue as its tangent direction at the critical point (as well as the sign in (2.5) reversed). One is interested in the unstable thimble because the coefficients $m_{\sigma}$ count the intersection of such thimbles with the original domain of integration, which in our case is the real axis (the sign ambiguity is not resolved by just this definition, but it can be deduced by means of other considerations). We have already pointed out that $S^{I}$ is constant along the thimble. However, one has to keep in mind the line element of the complex integral, that is

$$
z^{\prime}(t)=\left|z^{\prime}(t)\right| e^{i \phi(t)}
$$

We call $\phi(t)$ the "residual phase" and, while it is trivial to compute for 0-dimensional models, in principle it could become a source of a new sign problem for a realistic field theory; nevertheless we expect it to be quite smooth and there is already numerical evidence for this (see $[2,3]$ ).

\section{The 0 -dimensional $\phi^{4}$ theory}

We now apply the previous considerations to the study of the action

$$
S(\phi)=\frac{1}{2} \sigma \phi^{2}+\frac{1}{4} \lambda \phi^{4}
$$

with $\lambda \in \mathbb{R}^{+}$and $\sigma=\sigma_{R}+i \sigma_{I} \in \mathbb{C}$. This toy model was initially proposed as a test for complex Langevin simulations in [4]. For a recent and thorough study of complex Langevin dynamics of 
this model, the reader can refer to [5]. One peculiar feature of this model is that complex Langevin simulations display divergences for $\left\langle\phi^{n}\right\rangle$ with $n>4$ in a certain region of parameters. The thimble approach gives no divergences for any value of the parameters. However we will see how the sign of $\sigma_{R}$ affects the values of $m_{\sigma}$, thus rendering the thimble structure quite non-trivial.

Now we complexify the field by setting $\phi=x+i y$. We get

$$
\begin{gathered}
S^{R}=\frac{1}{2}\left[\sigma_{R}\left(x^{2}-y^{2}\right)-2 \sigma_{I} x y\right]+\frac{1}{4} \lambda\left(x^{4}+y^{4}-6 x^{2} y^{2}\right) \\
S^{I}=\frac{1}{2}\left[\sigma_{I}\left(x^{2}-y^{2}\right)+2 \sigma_{R} x y\right]+\lambda\left(x^{3} y-x y^{3}\right)
\end{gathered}
$$

from which the steepest ascent equations follow

$$
\begin{aligned}
& \frac{\partial S^{R}}{\partial x}=\sigma_{R} x-\sigma_{I} y+\lambda x^{3}-3 \lambda x y^{2} \\
& \frac{\partial S^{R}}{\partial y}=-\sigma_{R} y-\sigma_{I} x+\lambda y^{3}-3 \lambda x^{2} y
\end{aligned}
$$

The hessian is built from the second derivatives of $S^{R}$ and takes the form

$$
H(x, y)=\left(\begin{array}{cc}
\sigma_{R}+3 \lambda x^{2}-3 \lambda y^{2} & -\sigma_{I}-6 \lambda x y \\
-\sigma_{I}-6 \lambda x y & -\sigma_{R}-3 \lambda x^{2}+3 \lambda y^{2}
\end{array}\right)
$$

There are 3 critical points: $\phi_{0}=0$ and $\phi_{ \pm}= \pm \sqrt{-\frac{\sigma}{\lambda}}$ (which are the two, complex valued, "Higgs vacua"). As for the $m_{\sigma}$, the situation is quite different between the 3 cases $\sigma_{R}>0, \sigma_{R}=0$ and $\sigma_{R}<0$ : in each case we computed the stable and unstable thimble associated with each critical point with the procedure described above. Figures 1,2 and 3 show the results for the three cases.

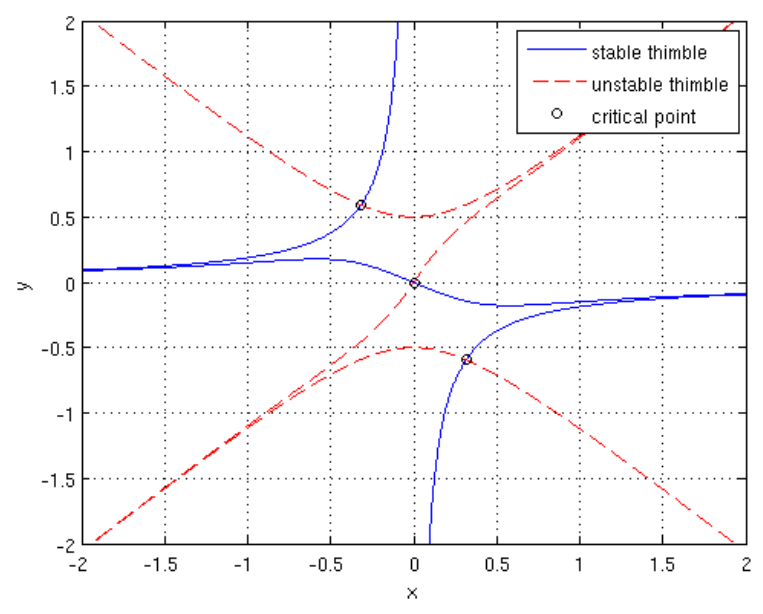

Figure 1: Stable and unstable thimbles associated with the three critical points for $\sigma=+0.5+0.75 i, \lambda=2$.

From Figure 1, we see that for $\sigma_{R}>0$ the unstable thimbles associated to the Higgs vacua do not intersect the real axis and therefore these points do not contribute to the integrals, that is $m_{ \pm}=0$. So, we must have $m_{0}=1$ and in fact with this assumption and by integrating along the 


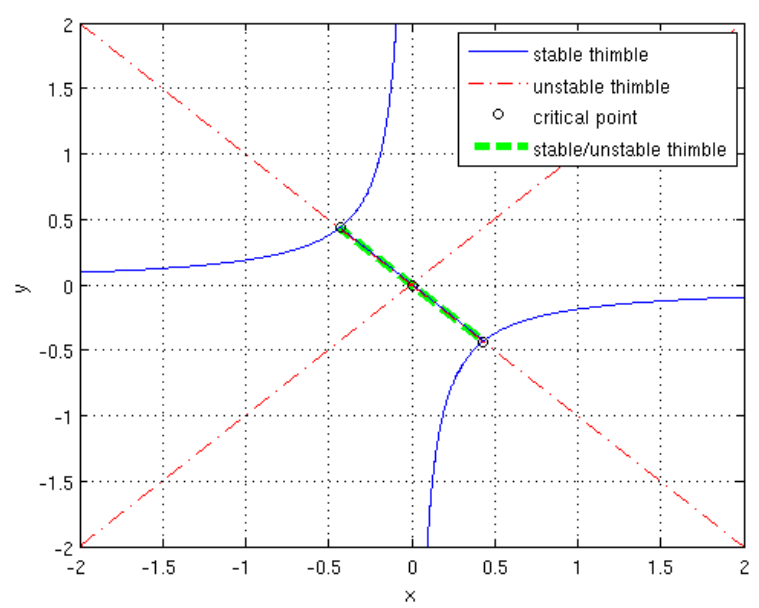

Figure 2: Stable and unstable thimbles associated with the three critical points for $\sigma=0.75 i, \lambda=2$.

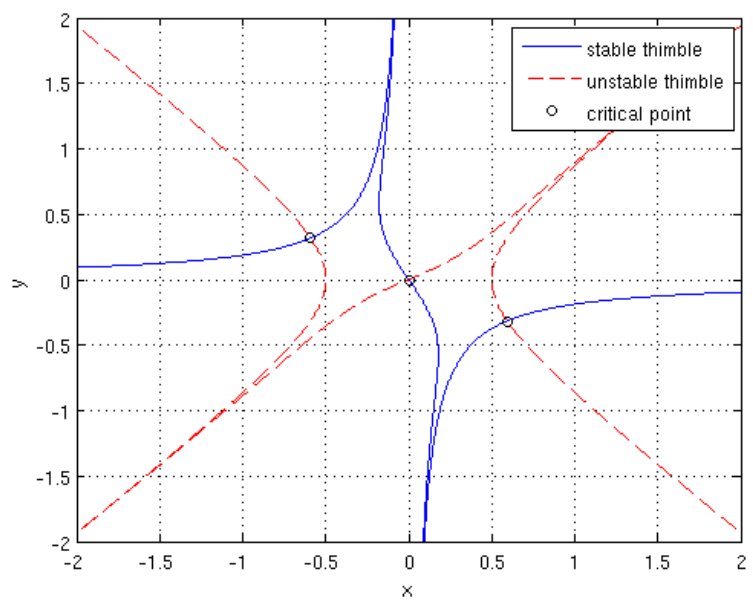

Figure 3: Stable and unstable thimbles associated with the three critical points for $\sigma=-0.5+0.75 i, \lambda=2$.

stable thimble attached to $\phi_{0}$, we recover the correct results for, say, $Z=\int e^{-S}$ (the integration can be easily carried on along the real axis both analytically and numerically, as the real part of the action grows very large with $x$ and oscillations are damped out). By decreasing $\sigma_{R}$ we can reach $\sigma_{R}=0$ : this situation, called "Stokes phenomenon", is depicted in Figure 2; for purely imaginary values of $\sigma$, the stable thimble connected to the critical point $\phi_{0}$ ends up into the Higgs vacua $\phi_{ \pm}$, so the action does not go to infinity as expected. The decomposition (2.3) does not hold in this case. The case $\sigma_{R}<0$ depicted in Figure 3 is a totally different matter. Now we see that the unstable thimbles connected to the Higgs vacua do intersect the real axis and therefore $m_{ \pm} \neq 0$, as well as $m_{0} \neq 0$. The correct combination which recovers the expected results for the integrals turns out to be $m_{0}=-1$ and $m_{ \pm}=+1$. These values were found by imposing continuity in the integral while crossing $\sigma_{R}=0$, which is guaranteed by the holomorphicity of the original action in the (complex) $\sigma$ parameter. 


\section{Monte Carlo simulations}

As pointed out before, we performed Monte Carlo simulation on the Lefschetz thimbles for this model. We tested different approaches: a Metropolis-like algorithm (described in [6]), a Langevinlike thimble Monte Carlo, that is the Aurora algorithm described in [7] (with the obvious trick that the tangent space to the thimble is locally given by $\nabla S^{R}$ ). We also applied a sort of "ideal sampling" along the thimble ([8]). All these methods lead to correct results for the observables $\left\langle\phi^{n}\right\rangle$. In Figure 4, for example, we see some results of our Metropolis simulations for computing $\left\langle\phi^{8}\right\rangle$ ( $\varepsilon$ is a technical parameter of the simulation - the smaller its value, the better the thimble is covered). In Figure 5 we see that we are able to cover the thimble with a stochastic integration process as well as we want.

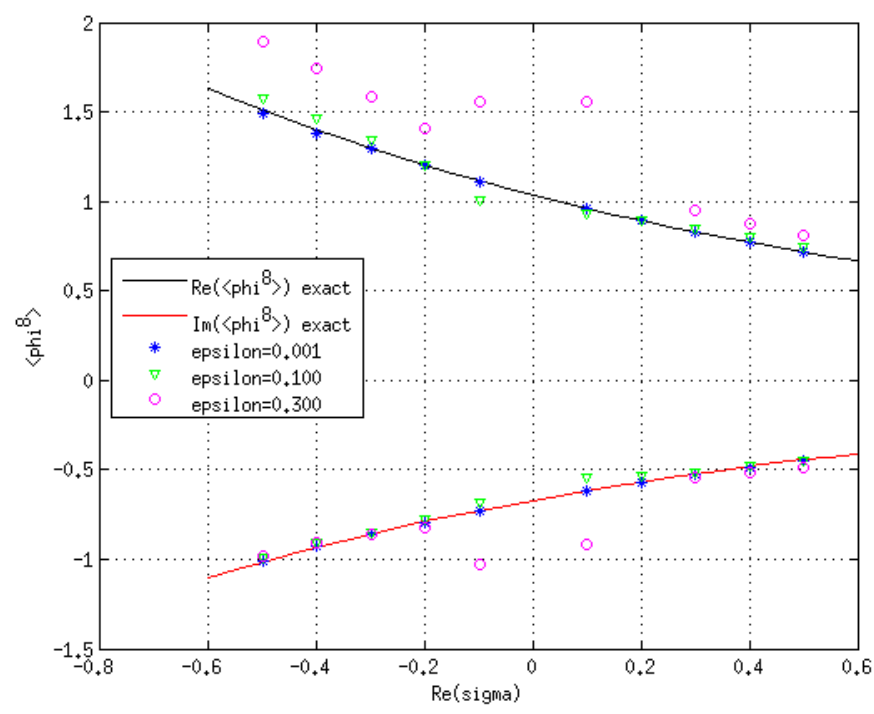

Figure 4: Metropolis Monte Carlo results for $\left\langle\phi^{8}\right\rangle$ with different values of $\sigma_{R}$.

\section{Conclusions}

We have discussed complex Morse theory applied to 0-dimensional toy models (which turn out to be extremely interesting in this respect), in particular to a case where complex Langevin failed to provide correct results in some regions of parameter space. We have also seen how different Monte Carlo can be devised to do importance sampling over Lefschetz thimbles, providing correct results. A generalization of what we have shown here to a general field theory is therefore a promising way to solve the sign problem (see [7]).

\section{Acknowledgements}

We thank L. Scorzato, M. Cristoforetti, A. Mukherjee, C. Torrero and C. Schmidt for precious discussions. 


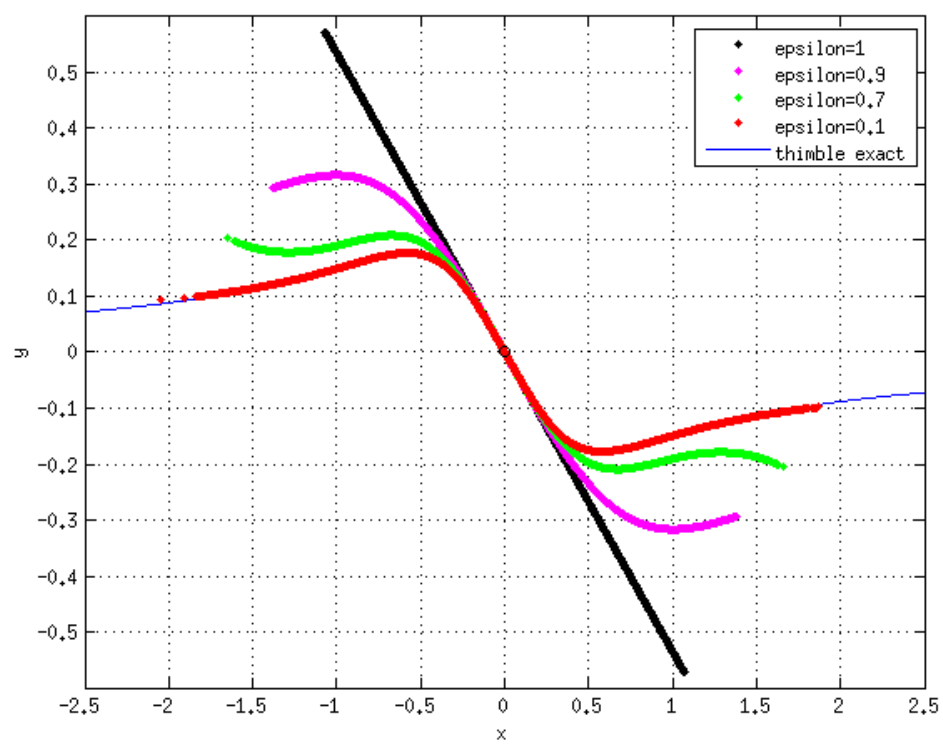

Figure 5: Thimble covering with Metropolis Monte Carlo results for $\sigma=+0.5+0.75 i, \lambda=2$.

\section{References}

[1] E. Witten, Analytic continuation of Chern-Simons Theory, arXiv:1001.2933v4 [hep-th].

[2] H. Fujii, D. Honda, M. Kato, Y. Kikukawa, S. Komatsu, T. Sano, Hybrid Monte Carlo on Lefschetz thimbles - A study of the residual sign problem, JHEP 1310 (2013) 147 [arXiv: 1309 . 4371v2] [hep-lat].

[3] M. Cristoforetti, F. Di Renzo, G. Eruzzi, A. Mukherjee, C. Schmidt, L. Scorzato, and C. Torrero, An efficient method to compute the residual phase on a Lefschetz thimble, Phys. Rev. D 89, 114505 (2014) [arXiv: 1403.5637v2] [hep-lat].

[4] J. Ambjørn, S. K. Yang, Numerical problems in applying the Langevin equation to complex effective actions, Phys. Lett. B 165 (1985) 140-146.

[5] G. Aarts, P. Giudice, E. Seiler, Localised distributions and criteria for correctness in complex Langevin dynamics, Annals Phys. Vol. 337 (2013) 238-260 [arXiv:1306.3075v1] [hep-lat].

[6] A. Mukherjee, M. Cristoforetti, L. Scorzato, Metropolis Monte Carlo on the Lefschetz thimble: application to a one-plaquette model, Phys. Rev. D 88, 051502(R) (2013) [arXiv: 1308 .0233v1] [physics.comp-ph].

[7] M. Cristoforetti, F. Di Renzo, L. Scorzato, New approach to the sign problem in quantum field theories: High density QCD on a Lefschetz thimble, Phys. Rev. D 86, 074506 (2012) [arXiv:1205.3996v3] [hep-lat].

[8] F. Di Renzo, An algorithm for thimble regularization of lattice field theories (and possibly not only for that), in proceedings of The 32nd International Symposium on Lattice Field Theory, POS (LATTICE2014) 046. 